\title{
Vandenboschia speciosa (Willd.) G. Kunkel en Jaizkibel: nuevas poblaciones con alta representación de su fase gametofítica y modelización de su distribución
}

\author{
Vandenboschia speciosa (Willd.) G. Kunkel Jaizkibelen: gametofito fasearen errepresen- \\ tazio handia duten populazio berrien aurkikuntza eta bere banaketaren modelizazioa
}

\author{
Vandenboschia speciosa (Willd.) G. Kunkel in Jaizkibel: new populations \\ with a high representation of their gametophytic phase and distribution model
}

\author{
Iñaki Sanz-Azkue ${ }^{1 *}$, Ibai Olariaga ${ }^{1}$, Ana Felipe ${ }^{2}$ \\ ${ }^{1}$ Sociedad de Ciencias Aranzadi-Aranzadi Zientzia Elkartea. Zorroagagaina,11. 20014 Donostia-San Sebastián. \\ 2E-mail: anafedi@gmail.com \\ *Correspondencia: isanz@aranzadi.eus
}

\section{RESUMEN}

Vandenboschia speciosa (Willd.) G. Kunkel es un helecho paleotropical que en el País Vasco habita rocas ácidas en zonas lluviosas y templadas. Es el único helecho europeo con fase gametofítica independiente y capaz de reproducirse vegetativamente mediante gemas. Cuenta con pocas poblaciones en Gipuzkoa y únicamente se conocían 3 cuadrículas con citas de esporofito en Jaizkibel. Prospecciones recientes en arroyos pertenecientes a los municipios de Pasaia y Hondarribia han proporcionado 7 nuevas localizaciones de esporofito, así como 30 más de gametofito, constatando que la mayor parte de individuos de las poblaciones corresponden a la fase gametofítica, pocas veces observada previamente tanto en el País Vasco como en la Península lbérica. En base a las localidades conocidas en Jaizkibel, se ha realizado una modelización de la distribución de $V$. speciosa en el macizo de Jaizkibel, hallándose nuevas zonas de alta probabilidad de presencia en las que se esperan encontrar nuevos individuos en prospecciones futuras. La cantidad de individuos conocidos hasta la fecha indica que el macizo de Jaizkibel es un área importante para la conservación y protección de este helecho amenazado.

PALABRAS CLAVE: Conservación, flora, lista roja, plantas amenazadas.

\section{LABURPENA}

Vandenboschia speciosa (Willd.) G. Kundel Euskal Herriko gune euritsu epeletan, arroka azidoetan, gune euritsuetan eta Euskal Heriko gune euritsu epeletan, arroka azidoetan bizi den iratze paleotropikala da. Gametofitoaren fase, belaunaldi independentea duen eta gema bidez begetatiboki haz daitekeen Europako iratze bakarra da. Gipuzkoan populazio gutxi ditu eta Jaizkibelen 1 x1 Km-ko 3 kuadrikula baino ez ziren ezagutzen egundaino. Pasai Donibane eta Hondarribiko udalerrietan egin berri diren behaketek, esporofitoaren 7 gune berri eta gametofitoaren 30 gune baino gehiago eman dituzte ezagutzera. Datu hauen arabera, populazioetako ale gehientsuenak gametofito aldian daude, duela gutxi arte Euskal Herrian eta lberiar Penintsulan gutxi aurkitu izan den fasean, hain zuzen ere. Jaizkibelen ezagutzera eman diren guneak kontuan izanik, mendikatean zehar espezieak edukiko lukeen zabalpenaren modelizazio lana egin da. Honela, iratzea aurkitzeko probabilitate altua duten gune berriak aurkitu ahal izan dira eta etorkizunera begira, ale gehiago aurkitzeko esperantza piztu da. Dena den, eta egundaino ezagutzen diren aleak kontuan izanik, Jaizkibel iratze mehatxatu honen kontserbaziorako eta babeserako gune garrantzitsua dela adieraz daiteke.

GAKO-HITZAK: Kontserbazioa, flora, zerrenda gorria, landare mehatxatuak.

\section{ABSTRACT}

Vandenboschia speciosa (Willd.) G. Kunkel is a paleotropical fern that inhabits acid rocks in warm rainy areas of the Basque Country. It is unique among European ferns due to independent gametophyte generation that produces gemae for vegetative reproduction. Vandenboschia speciosa has few populations in Gipuzkoa, and only $31 \times 1 \mathrm{~km}$ squares were known from Jaizkibel. Fieldwork undertaken in Pasaia and Hondarribia revealed the presence of 7 new sites of sporophytes and more than 30 new gametophyte sites. According to our observations, most of the individuals of the populations correspond to gametophytes, even though gametophytes have been few times reported until recent in the Basque Country and the Iberian Peninsula. A model has been constructed to predict the distribution of this fern in Jaizkibel, inferred from all the new reports. The model shows new sites where the presence of $V$. speciosa is very probable and that need to be prospected in the future. Judging from the amount of $V$. speciosa specimens hitherto reported, Jaizkibel is an important area for its conservation and protection.

KEY WORDS: Conservation, flora, red list, threatened plants.

\section{INTRODUCCIÓN}

Vandenboschia speciosa (Willd.) G. Kunkel (Hymenophyllaceae) es un helecho paleotropical de distribución macaronésica que vive en rocas ácidas, en lugares umbríos y alto grado de humedad (Ratcliffe et al., 1993; Rumsey, 1994; Pérez-Latorre et al., 2005). La incapacidad de retención del agua de su fase esporofítica hace que $V$. speciosa sea un helecho muy sensible a la sequía (Rumsey, 1994), que requiera de hábitats de humedad constante y temperatura poco variable, con inviernos suaves y pocas heladas (Ratcliffe et al., 1993). Estos factores influyen en que este helecho se distribuya principalmente por la costa atlántica europea (Boudrie, 1998; Sociedad de 
Ciencias Naturales de Sestao, 2001), en zonas cercanas a regatas (Felipe-Díaz et al., 2012). Su fase gametofítica, independiente de la esporofítica, no posee tales requerimientos ecológicos (Johnson et al., 2000; Macgomol \& Sheffield, 2001), por lo que se conocen poblaciones gametofíticas de $V$. speciosa en el centro de Europa (Krippel, 2001; Krukowski \& Swierkosz, 2005) en zonas con condiciones en las que el esporofito no puede subsistir. Todas estas características hacen que este helecho se encuentre catalogado como vulnerable en el Catálogo Vasco de especies amenazadas y en la Lista Roja de la Flora Vascular de la Comunidad Autónoma del País Vasco (CAPV) y está incluido en el Anexo II de la Directiva Hábitat (Aizpuru et al., 2010).

Jaizkibel, con pequeñas regatas de corta distancia orientadas al N-NO, que nacen en el mismo macizo y desembocan en el mar, resulta un lugar de potencialidad alta para la presencia de la especie. Sus regatas encajonadas, protegidas muchas veces por bosque de ribera, con pequeñas cascadas y abundantes afloramientos de rocas de arenisca con oquedades, permiten la presencia del gametofito y del esporofito a lo largo de todo el macizo.

Las primeras citas de $V$. speciosa en Jaizkibel realizadas a finales del siglo XIX por Gandoger (1898) fueron, a su vez, la primera observación del helecho para el País Vasco. Aunque no se sabe la localización exacta de su cita, Gandoger afirma haber visto la especie en las "grutas húmedas de Jaizkibel", tanto en la zona de Pasaia como en la de Hondarribia (Gandoger, 1898). Unos pocos años más tarde, a principios del siglo XX, pteridólogos franceses publicaron más observaciones del helecho en el macizo (Zeiller, 1905; Litardiére, 1913). Desde entonces, las últimas citas corresponden a los años 80 (González \& Fernández de Pinedo, 1980; Aseginolaza et al., 1984; Catalán \& Aizpuru, 1984; Catalán \& Aizpuru, 1985).

Todas estas observaciones corresponden a esporofitos, ya que hasta el año 2010 se desconocía la presencia del gametofito en la Comunidad Autónoma Vasca (Sanz-Azkue et al., 2010), aunque ya se había citado anteriormente a pocos kilómetros, en las regatas Lizarlan y Lantzeta, en la localidad labortana de Biriatu (Jovet, 1933; Loriot, 2005).

Los últimos estudios realizados con $V$. speciosa en el País Vasco, demuestran que la presencia del gametofito es mucho más abundante de lo pensado (Loriot, 2005; Oreja et al., 2008; Garmendia \& Oreja, 2012; SanzAzkue et al., 2013) y así lo demuestra el presente trabajo, para el que se ha realizado un muestreo específico de la especie en Jaizkibel.

Dado que la detección y contabilización de individuos de $V$. speciosa es esencial para valorar el estado de conservación de las especie en el País Vasco (SanzAzkue et al., 2010), en este trabajo, además del muestreo de campo, se realiza una modelización de la distribución potencial del helecho en Jaizkibel, con el objetivo de descubrir nuevos individuos de la especie y optimizar el esfuerzo de muestreo.

\section{MATERIAL Y MÉTODOS}

Inicialmente, se realizó una exhaustiva búsqueda bibliográfica para determinar el número de citas conocidas de $V$. speciosa en el macizo de Jaizkibel. A su vez, con el mismo fin, se ha consultado el herbario ARAN de la Sociedad de Ciencias Aranzadi y se han recogido citas más recientes recopiladas por Y. Meyer (Ekos S.L.), J. Emparan y A. Alkain. Una vez recopiladas las observaciones, se ha querido comprobar la existencia actual de dichas poblaciones, para lo que se han muestreado las cuadrículas de $1 \times 1 \mathrm{~km}$ a las que pertenecen.

Una vez finalizada la búsqueda bibliográfica, se realizó el trabajo de campo entre May. y Nov. de 2012, con el fin de de encontrar nuevos individuos en su fase esporofítica como gametofítica en Jaizkibel. La búsqueda se hizo en parte de 14 regatas del municipio de Hondarribia orientadas al N, NO y una perteneciente al municipio de Pasaia. Las coordenadas se recogieron con un GPS, con un error de entre 5 y $10 \mathrm{~m}$.

Una vez recopiladas todas las observaciones, antiguas y recientes, se realizó un estudio de modelización del helecho en el macizo. La modelización se efectuó en base a 37 coordenadas de esporofitos y gametofitos, empleando el programa MAXENT 3.3.3.k. Este modelo está basado en el principio de máxima entropía y entre sus principales ventajas se encuentran: (1) requiere solo de datos de presencia, en conjunto con variables ambientales; (2) puede usar tanto datos continuos como categóricos; (3) es aplicable a múltiples escalas y campos; (4) utiliza eficientes algoritmos en continua renovación (Phillips \& Dudík, 2008); (5) es un modelo generativo, no discriminativo, lo cual es ventajoso especialmente con un número pequeño de muestras (Elith et al., 2006). Se optó por modelar conjuntamente ambas fases del ciclo, dado que en Jaizkibel ambos comparten el mismo hábitat, aunque tienen distintos requerimientos de microhábitat. El hecho de que la modelización no se pueda hacer a nivel de microhábitat y el bajo número de citas de las que disponíamos (no se han encontrado más), hizo que se optara por esa opción, ya que este programa puede ser sensible a la cantidad y distribución espacial de los datos de origen (Elith \& Leathwick, 2009).

Se utilizaron 7 variables ambientales para su implementación: distancia a ríos, distancia al mar, hábitats según la clasificación de los tipos de hábitat de EUNIS, orientación, MDT, litología y pendiente. Las variables de distancia a ríos y distancia a mar fueron generadas a partir de la cartografía hidrográfica y la línea de costa respectivamente, utilizando la herramienta Straight Line de Arcgis. El resto de las variables se obtuvieron de la cartografía de uso libre del Gobierno Vasco (ftp://ftp.geo.euskadi.net).

Para su utilización en Maxent, todas las variables deben tener la misma extensión geográfica y tamaño de píxel. Por tanto, aunque la escala de origen de las variables no coincidiera, se ajustaron todas las capas ráster para que tuvieran una resolución espacial de $5 \mathrm{~m}$. 
Se realizaron 10 réplicas del modelo para obtener el resultado final, empleándose alrededor del $80 \%$ de los puntos para construir el modelo y el $20 \%$ de los registros restantes como puntos de validación. Para estimar la capacidad predictiva del modelo se usó el test estadístico AUC a partir de la curva ROC. Asimismo se utilizó la prueba Jackknife para medir la importancia de cada variable.

\section{RESULTADOS}

\section{Búsqueda bibliográfica}

La búsqueda de citas previas en el herbario ARAN y en la bibliografía dio como resultado un total de 5 puntos diferentes en los que se conocía la existencia de la especie en Jaizkibel. Sin embargo, dos de las citas bibliográficas más antiguas de finales del siglo XIX y comienzos del XX únicamente precisaban el municipio (Gandoger, 1898; Zeiller, 1905), imposibilitando su búsqueda exacta. Litardière (1913), daba a su vez observaciones con una precisión de $1 \times 1 \mathrm{~km}$, pero con coordenadas que no pertenecían al macizo, por lo que se decidió no tener en cuenta dicha observación. Así, en total se tuvieron en consideración tres cuadrículas de $1 \times 1 \mathrm{~km}$ de observaciones realizadas en los años 80, las cuales posterior hemos podido constatar que siguen presentes en el macizo.

\section{Muestreos}

Estas mismas cuadrículas y algunas más pertenecientes a diferentes regatas, fueron posteriormente mues- treadas. De las 15 regatas prospectadas, en 12 (80\%) se ha encontrado el helecho en alguna de las dos fases o en algunos casos, tanto en su fase gametofítica como en la esporofítica. En tres de las regatas (Aizporaundi, Mendizorrotz y Mariputzu) no se ha encontrado la especie, aunque no se descarta su presencia (Tabla 1).

Gracias a los muestreos realizados, el número de cuadrículas $1 \times 1 \mathrm{~km}$ con presencia de la especie en Jaizkibel ha pasado de 3 cuadrículas conocidas a un total de 14 (Fig. 1). La presencia del helecho en fase esporofítica

\begin{tabular}{l|c|c|}
\hline \begin{tabular}{l|c|} 
Regata \\
HONDARRIBIA
\end{tabular} & $\mathbf{N}^{\circ}$ de núcleos de EF & $\mathbf{N}^{\circ}$ de rocas con GF \\
\hline Aizporaundi erreka & 0 & 0 \\
\hline Enekuzko erreka & 0 & $0-5$ \\
\hline Mendizorrotz erreka & 0 & 0 \\
\hline Kapeluetako erreka & 1 & $0-5$ \\
\hline Martierreka & 2 & $0-5$ \\
\hline Mariputzuko erreka & 0 & 0 \\
\hline Erramudi erreka & $>2$ & $>10$ \\
\hline Iturraingo erreka & 0 & $>10$ \\
\hline Txortxipi erreka & 1 & $5-10$ \\
\hline Martitxerreka & 0 & $0-5$ \\
\hline Mintegiko erreka & 1 & 0 \\
\hline Agerdi erreka & 1 & $5-10$ \\
\hline Punta Turrilla & 1 & $0-5$ \\
\hline Gaztarrotz erreka* & 0 & $5-10$ \\
\hline PASAIA & 1 & \\
\hline Marguzes erreka & \\
*Comparte municipio con & & \\
\hline
\end{tabular}

Tabla 1. - Número de núcleos de esporofito y rocas con gametofito encontrados durante los muestreos en el macizo de Jaizkibel.

1. Taula. Jaizkibelen eginiko laginketetan aurkituriko esporofito nukleo kopurua eta gametofitodun harkaitz kopurua.

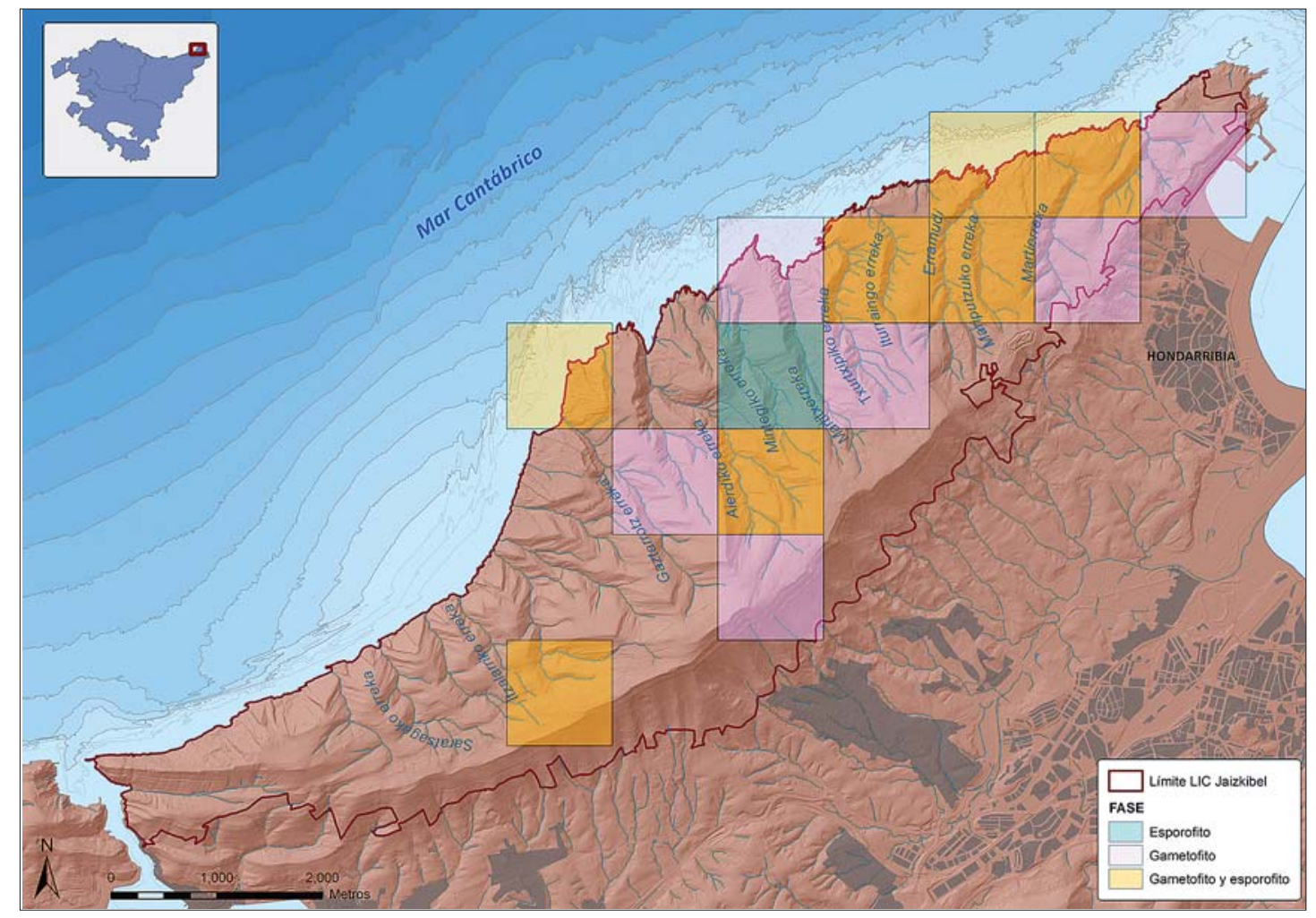

Fig. 1. - Cuadrículas UTM $1 \times 1 \mathrm{~km}$ con presencia de $V$. speciosa en el macizo de Jaizkibel. 1. Irudia. Jaizkibelen V. Speciosa espeziearen presentzia ezaguna den $1 \times 1 \mathrm{~km}-\mathrm{ko}$ UTM kuadrikulak. 
(Fig. 2) ha incrementado de 3 cuadrículas a 8, con cinco nuevas, de entre las cuales, en la regata de Martierreka, se han localizado dos núcleos dentro de la misma cuadrícula. En cuanto al gametofito se refiere, éste ha sido citado por primera vez en el macizo de Jaizkibel y se ha constatado su presencia en 13 nuevas cuadrículas (Fig. 1) y más de 30 localizaciones no conocidas anteriormente. Destaca, a su vez, la presencia del gametófito en todas las cuadrículas en las que se ha encontrado el esporofito, excepto en la regata Mintegi (Y. Meyer, com. pers.) (Fig. 1), en la que no se ha podido realizar aún ninguna prospección para la búsqueda de la fase gametofítica.

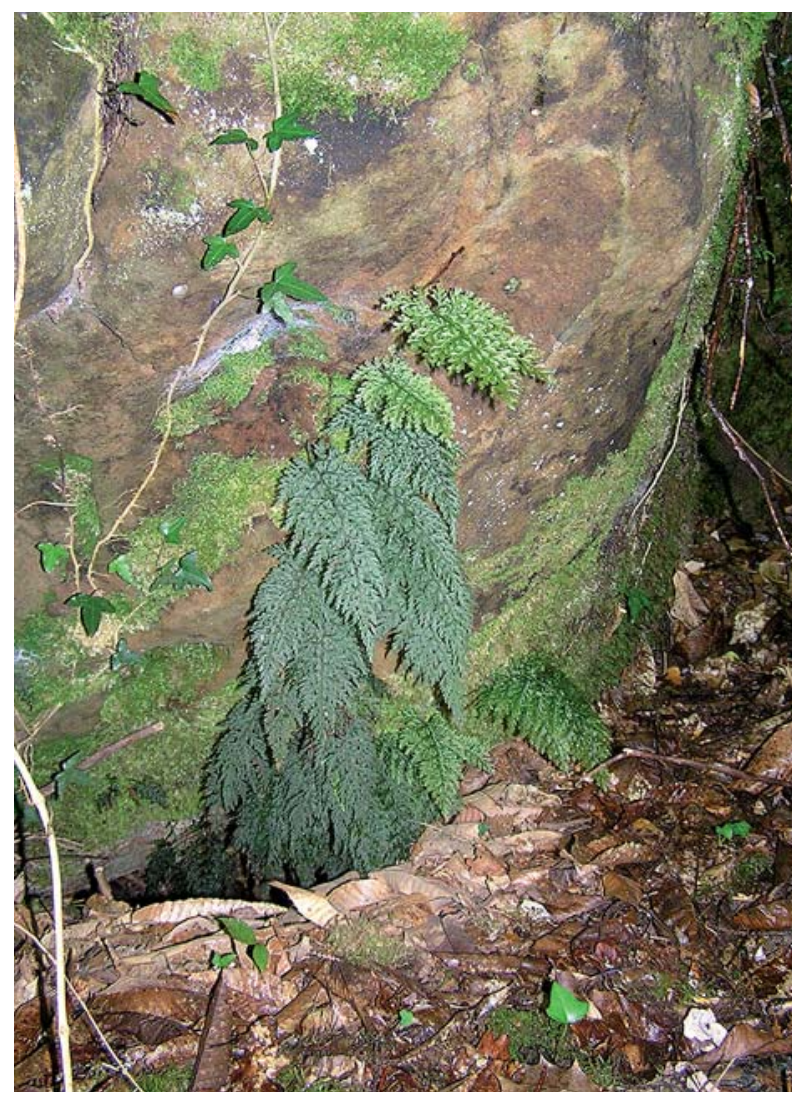

Fig. 2. - Esporofito de V. speciosa en la regata Martierreka de Hondarribia. Foto: I. Sanz-Azkue.

2. Irudia. V. speciosa-ren esporofitoa Martierrekan. Arg.: I. Sanz-Azkue

En cuanto a los ríos se refiere, destaca la regata Erramudi con dos núcleos de esporofito, uno de ellos con varias manchas de frondes separadas varios metros cuadrados, y varios núcleos con gametofitos encontrados a lo largo de toda la regata en oquedades de afloramientos de roca de arenisca. También destaca la presencia en una zona concreta de la regata de manchas de gametofito de gran envergadura, en el que se pueden observar varios brotes de pequeños esporofitos (Fig. 3).

\section{Modelización}

La prueba de Jackknife sirvió para medir la importancia de cada variable (Fig. 4). El porcentaje de contri-

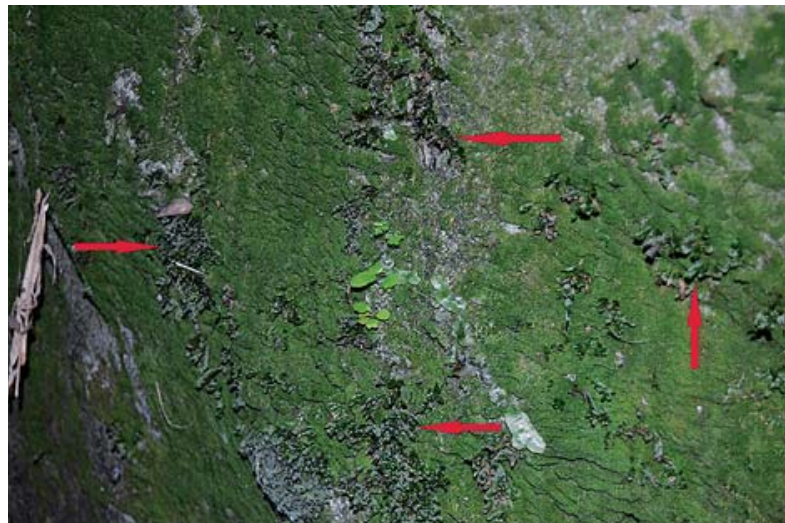

Fig. 3. - Pequeños brotes de esporofitos repartidos por una roca de arenisca cubierta por el gametofito de V. speciosa. Foto: M. Nieto.

3. Irudia. $V$. speciosa-ren gametofitoaz estalitako hareharrian barreiatzen diren esporofitoaren landare txikiak. Arg.: M. Nieto.

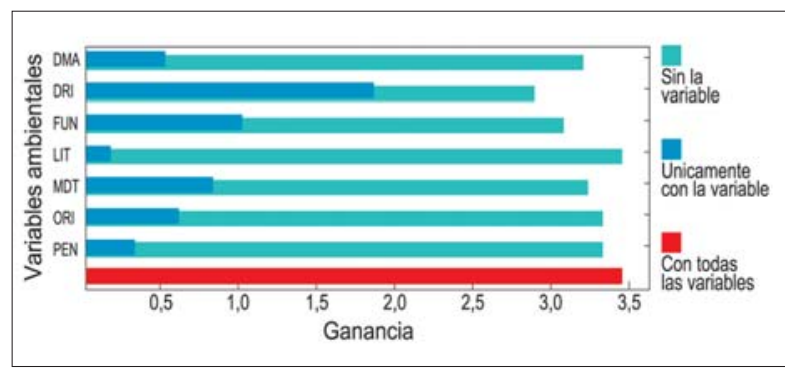

Fig. 4.- Prueba Jackknife para la ganancia de los datos de entrenamiento (DMA: Distancia al mar, DRI: Distancia al curso de agua más próximo, EUN: Hábitats EUNIS, LIT: Litología, MDT: Modelo Digital del Terreno, ORI: Orientación, PEN: Pendiente).

4. Irudia. Jackknife froga entrenamendurako datuen irabazirako (DMA: Itsasora distantzia, DRI: Gertueneko errekara distantzia, EUN: EUNIS habitatak, LIT: Litologia, MDT: Lurraren Modelo Digitala, ORI: Orientazioa, PEN: Malda).

bución relativa de las variables en la distribución de $V$. speciosa (Tabla 2) muestra la importancia de la distancia a los ríos y del hábitat EUNIS en la distribución de la especie. No es de extrañar, dado que los requerimientos ecológicos de ambas especies están totalmente relacionados con zonas húmedas con temperaturas constantes que por otro lado, las encuentran en regatas con abundante bosque de ribera.

El valor medio de 0.988 (AUC) obtenido en la curva ROC (Fig. 5) corrobora el excelente ajuste del modelo para la especie estudiada con las variables empleadas.

Por otro lado, el trabajo de modelización realizado para conocer la potencialidad de las regatas de Jaizkibel para albergar núcleos de $V$. speciosa ha dado como re-

\begin{tabular}{|l|c|}
\hline Variable & Contribución (\%) \\
\hline Distancia a ríos & 48,6 \\
\hline Hábitats EUNIS & 21,9 \\
\hline Distancia a mar & 11,0 \\
\hline Orientación & 6,9 \\
\hline MDT & 6,2 \\
\hline Pendiente & 4,3 \\
\hline Litología & 1,2 \\
\hline
\end{tabular}

Tabla 2. - Porcentaje de contribución relativa de las variables en el modelo de distribución de $V$. speciosa. 2. Taula. Aldagaien ekarpen erlatiboaren portzentaia V. speciosaren zabalpenaren *Modelo Digital del Terreno. 


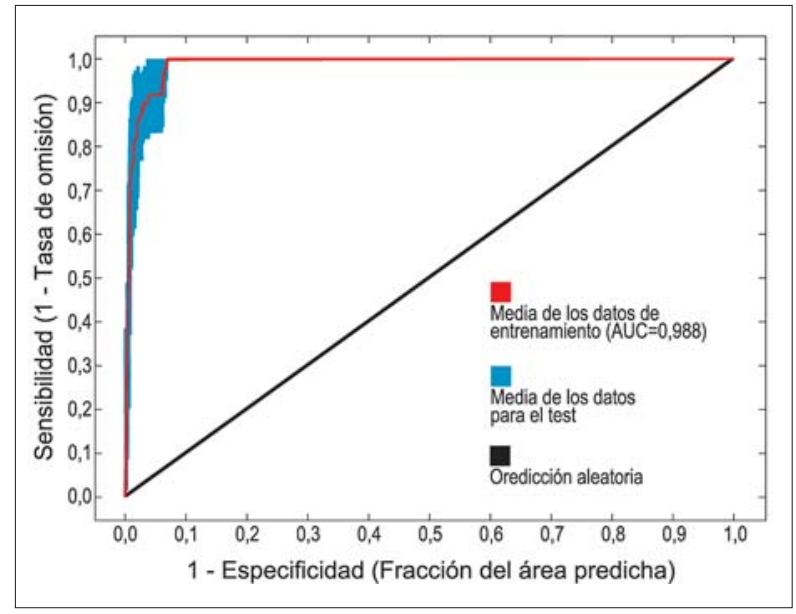

Fig. 5. - Curva de la tasa de omisión y área predicha.

5. Irudia. Baztertze-tasaren eta iragarpen azaleraren kurba.

sultado un mapa en el que se observa una clara división entre la ladera sur, con una idoneidad muy baja y la ladera norte, que da al mar, con una potencialidad mucho más alta para la especie (Fig. 6). Entre estas últimas, son las regatas de Hondarribia las que mejores condiciones presentan, mientras que las regatas pertenecientes a Pasaia Donibane, mucho más abiertas, con menos vegetación de ribera y con orientación $\mathrm{NO}$, en general no reúnen condi- ciones generales tan buenas como las anteriormente mencionadas, aunque sí se observan zonas en las que la disponibilidad de hábitat es relativamente alta (Fig. 6).

Sin embargo, no se debe olvidar que $V$. speciosa es una especie muy dependiente al microhábitat en el que vive (Felipe-Díaz et al., 2012). Por ello, pueden darse casos en los que la regata presenta condiciones generales muy buenas pero que la ausencia de buenos microhábitats para la especie haga que esté ausente en dicho lugar. O al contrario, puede darse el caso en el que una zona con condiciones generales bajas alberge la especie dado que sus características de microhábitat facilitan su presencia. Este último caso, se ha dado en regatas como Martitxerreka o Marguzes, en el que en zonas muy abiertas, expuestas al sol, sin apenas bosque de ribera y en zonas con poca potencialidad para la especie, se ha encontrado el gametofito en oquedades oscuras (Fig. 7) y húmedas de afloramientos de arenisca que hacían que la presencia de la especie fuera posible.

\section{DISCUSIÓN}

Los muestreos de campo indican que el número de individuos y localizaciones de $V$. speciosa en Jaizkibel es considerablemente más alto de lo previamente estimado. De 3 cuadrículas conocidas hasta el presente, se ha pasado a un total de 14 cuadrículas $1 \times 1 \mathrm{~km}$ en el que se co-

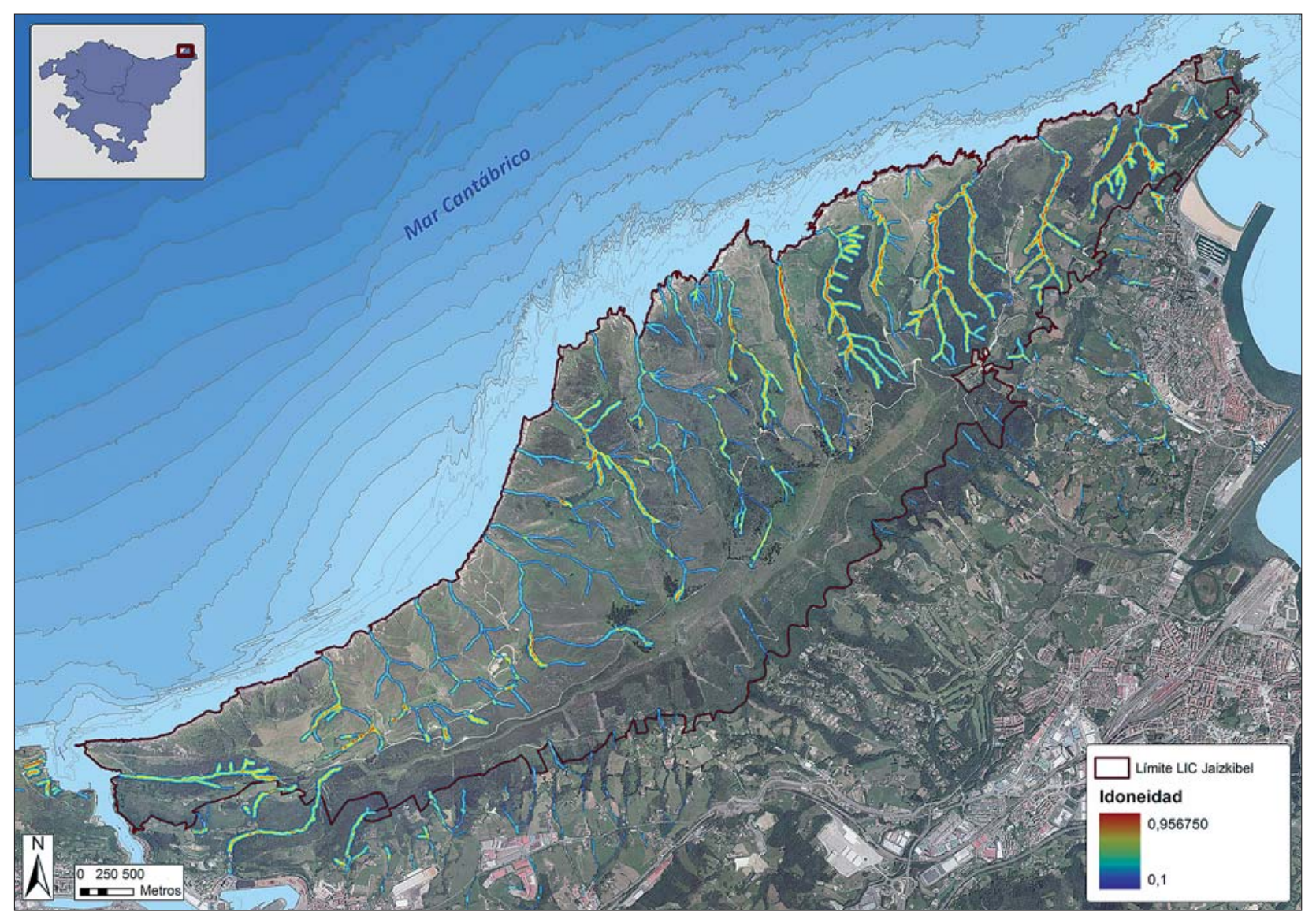

Fig. 6. - Idoneidad de las regatas de Jaizkibel para la presencia de $V$. speciosa.

6. Irudia. V. speciosa iratzea aurkitzeko Jaizkibelgo erreken egokitasuna 


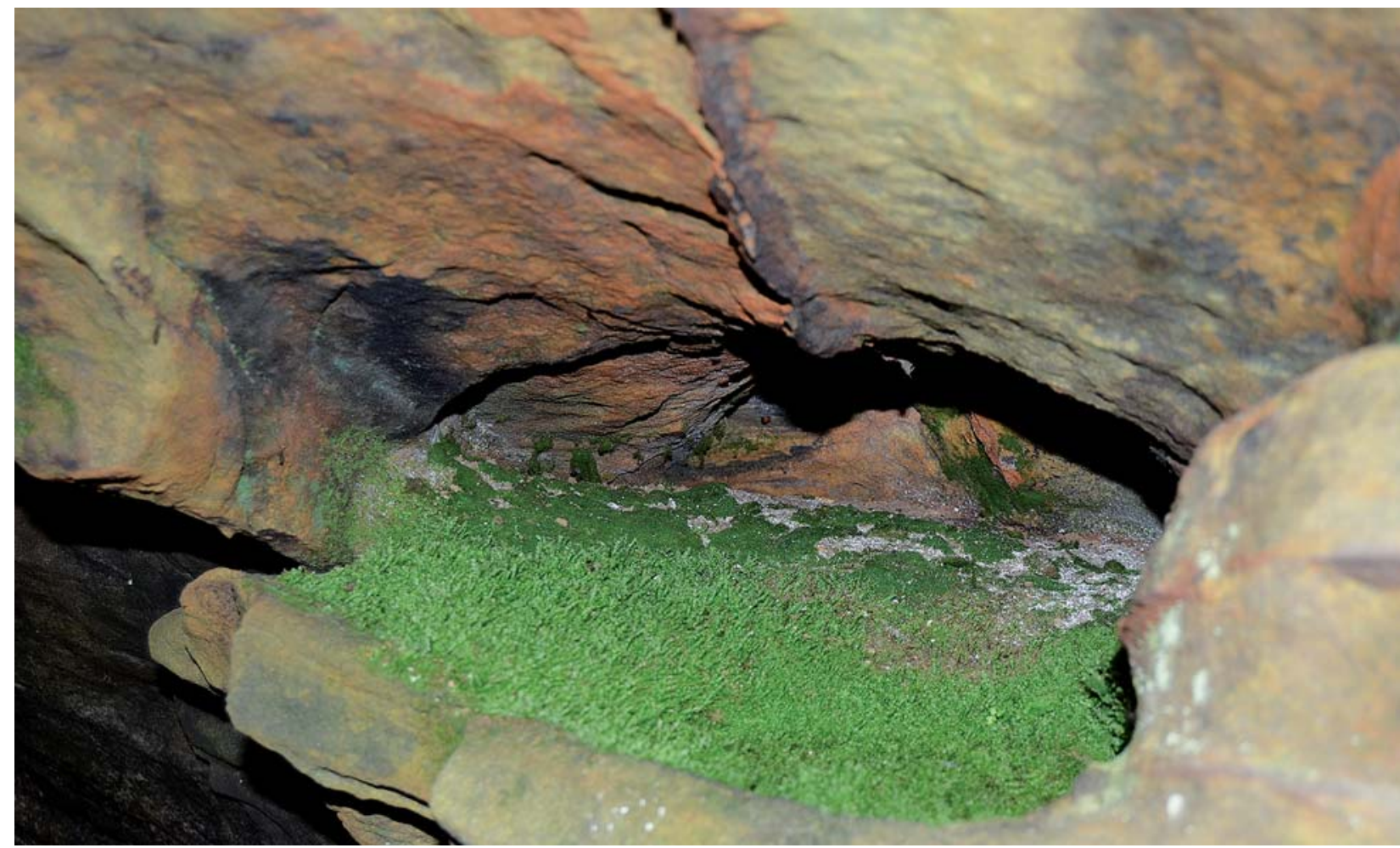

Fig. 7. - Gametofito de $V$. speciosa en el fondo de una oquedad de una roca de arenisca en la regata Iturrain de Hondarribia. Foto: M. Nieto.

7. Irudia. V. speciosa-ren gametofitoa hareharrizko arroka bateko zuloaren hondoan Iturrain errekan. Arg.: M. Nieto.

noce la presencia de la especie. A su vez, las observaciones realizadas demuestran que la mayor parte de los individuos de las poblaciones de Jaizkibel residen en forma de gametofito, con un total de 30 localizaciones repartidas en 13 cuadrículas. El gametofito, muy dependiente a las condiciones del microhábitat, se encuentra principalmente en las zonas más oscuras de oquedades de afloramientos de arenisca cercanas a las regatas. El esporofito, menos frecuente en el macizo, está distribuido en 8 cuadrículas, con 9 localizaciones.

El modelo de distribución potencial obtenido a partir de los datos de presencia de la especie en las dos fases, revela la presencia de zonas de potencialidad alta (Fig. 6), lo que hace pensar que el número de individuos que habita en Jaizkibel es aún mayor. Las variables con mayor importancia relativa en el modelo fueron la distancia a ríos y los hábitats EUNIS. Por consiguiente, varias regatas han de ser prospectadas en el futuro para comprobar la idoneidad del modelo, así como para cuantificar y cartografiar en detalle las poblaciones de $V$. speciosa en Jaizkibel, con sus consiguientes implicaciones en materias de conservación y posibles amenazas para su subsistencia.

\section{AGRADECIMIENTOS}

A Víctor Suárez, Marta Nieto y Samira Ben-Menni de la Univ. de Granada y a Iñaki Aizpuru por la ayuda prestada en la realización de los muestreos. A Xabier Lizaur, Yves Meyer, Joserra Emparan y Angel Alkain por la ayuda prestada en la localización de citas del esporofito de V. speciosa.

\section{BIBLIOGRAFÍA}

Aizpuru, I., Tamaio, I., Uribe-Echebarría, P.M., Garmendia, J., Oreja, L., Balentzia, J., Patino, S., Prieto, A., Biurrun, I., Campos, j. A., García, I., Herrera, M. 2010. Lista Roja de la Flora Vascular de la CAPV. Eusko Jaurlaritza-Gobierno Vasco. Vitoria-Gasteiz.

Aseginolaza, C., Gomez, D., Lizaur, X., Montserrat, G. 1984. Catalogo Florístico de Alava, Vizcaya y Guipúzcoa. Gobierno Vasco-Eusko Jaurlaritza. Vitoria-Gasteiz.

Boudrie, M. 1998. Les ptéridophytes du Pays Basque et du nordouest de l'espagne; écologie, répartition, protection. J. Bot. Soc. Bot. Fr. 5: 43-52.

Catalán, P., Aizpuru, I. 1984. Pteridófitos del monte Jaizkibel (Guipúzcoa). An. Biol. Univ. Murcia 1: 253-259

Catalán, P., Aizpuru, I. 1985. Aportación al catálogo florístico de la cuenca del Bidasoa (Guipúzcoa y Navarra). Munibe, Cienc. Nat. 37:17-86

Elith, J., Graham, C., Anderson, R., Dudík, M., Ferrier, S., Guisan, A., Hijmans, R., Huettmann, F., Leathwick, J., Lehmann, A., et al. 2006. Novel methods improve prediction of species' distributions from occurrence data. Ecography 29: 129-151.

Elith, J., Leathwick, J.R. 2009. Species Distribution Models: Ecological Explanation and Prediction Across Space and Time. Annu. Rev. Ecol. Evol. Syst. 40: 677-697.

Felipe-Díaz, A., Olariaga, I., Sanz-Azkue, I. 2012. Trichomanes speciosum iratzearen banaketa potentzialaren analisia Hernanin eta Urumea arroan. Hernaniko Udala - Ayuntamiento de Hernani.

Gandoger, M. 1898. Notes sur la flore espagnole. Bull. Soc. Bot. Fr. 45: 17-29.

Garmendia, J., Oreja, L. 2012. Información sobre el estado de conservación de Vandenboschia speciosa (Willd.) Kunkel en Na- 
varra para la elaboración del informe del artículo 17 de la Directiva Hábitat. Informe inédito elabora por la S.C. Aranzadi para Gestión Ambiental, Viveros y Repoblaciones de Navarra.

Gonzalez, E., Fernandez De Pinedo, M.C., 1980. Estudio de las repercusiones ecológicas de las plantaciones de Coníferas sobre la vegetación natural del País Vasco. En: Estudio ecológico y económico de las repoblaciones de Coníferas Exóticas en el País Vasco. Sociedad de Ciencias Aranzadi (Ed.): 432-609. Caja Laboral Popular. Mondragón (Guipúzcoa).

Johnson, G.N., Rumsey, F.J., Headley, A.D., Sheffield, E. 2000. Adaptations to extreme low light in the fern Trichomanes speciosum. New Phytologist 148: 423-431.

Jovet P. 1933. Le Trichomanes radicans (Sw.) et l'Hymenophyllum tunbridgense (Sm.) en Pays Basque français. Bull. Soc. Bot. Fr. 80: $797-809$

Krippel, Y. 2001. Aire de répartition et statut de Trichomanes speciosum Willd. (Hymenophyllaceae) au Luxembourg. Bull. Soc. Nat. Luxemb. 102: 3-13.

Krukowski, M., Swierkosz, K. 2004. Discovery of the gametophytes of Trichomanes speciosum (Hymenophyllaceae: Pteridophyta) in Poland and its biogeographic importance. Fern Gaz. 17(2): 79-85.

Litardière, R. de. 1913. Quelques herborisations en Pays Basque. Bull. Biarritz-Association. 18: 388-391.

Loriot, S. 2005. Pour une stratégie de conservation de Trichomanes speciosum Willd. dans le Massif Armoricain. Thesis doctorel. Université de Brest.

Macgomol, K., Sheffield, E. 2001. Gametophyte morphology and ultrastructure of the extremely deep shade fern, Trichomanes speciosum. New Phytol. 151: 243-255.
Oreja L., Berastegui, A., Arbelaitz, E., Garmendia, J., Urkizu, A. Tamayo, I. 2008. Diagnóstico del estado de conservación y propuestas de gestión de Vandenboschia speciosa (Willd.) Kunkel en Navarra. Gobierno de Navarra.

Pérez-Latorre, A.V., Cano, M.J., Cabezudo, B., Guerra, J. 2005 Phytocoenological behaviour, distribution and conservation of Trichomanes speciosum Willd. (Pteridophyta) in the south of the Iberian Peninsula. Cryptogam. Bryol. 26 (3): 249-261.

Phillips, S.J., Dudík, M. 2008. Modeling of species distributions with Maxent: New extensions and a comprehensive evaluation. Ecography 31: 161-175.

Ratcliffe, D.A., Birks, H.J.B., Birks, H.H. 1993. The ecology and conservation of the Killarney fern Trichomanes speciosum Willd. in Britain and Ireland. Biol. Conserv. 66: 231-247.

Rumsey, F.J. 1994. The distribution and population biology of the Killarney fern (Trichomanes speciosum Willd.). Unpublished Phd Tesis. University of Manchester.

Sanz-Azkue, I., Olariaga, I., Díez, J. 2010. Hernaniko landare mehatxatuen mikroerreserben sarea. Hernaniko Udala-Ayuntamiento de Hernani.

Sanz-Azkue, I., Olariaga, I., Díez López, J.R. 2013. Inventory and mapping of Red-listed Vascular Flora in Hernani municipality (GlPUZKOA, Basque Country). Munibe, Cien. Nat. 61:7-32.

Sociedad de Ciencias Naturales de Sestao. 2001. Helechos paleotropicales de la Comunidad Autónoma Vasca. Departamento de Agricultura y Pesca del Gobierno Vasco.

Zeiller, R. 1905. Sur la découverte de stations nouvelles du Trichomanes radicans dans les Basses-Pyrénées. Bull. Soc. Bot. Fr. 52: 65-67. 